\title{
Pattern formation and directional and spatial ordering of edge dislocations in bulk GaN: Microphotoluminescence spectra and continuum elastic calculations
}

\author{
Nikolaus Gmeinwieser and Ulrich T. Schwarz* \\ Department of Physics, University of Regensburg, D-93040 Regensburg, Germany \\ (Received 14 March 2007; revised manuscript received 17 April 2007; published 27 June 2007)
}

\begin{abstract}
We present a detailed microphotoluminescence study of the long-range strain fields surrounding threading dislocations as well as their interaction and pattern formation in GaN bulk crystals. The stress patterns are detected by tiny energy shifts of the near-band-edge spectral lines and show a dipolelike stress state around the dislocation core of edge- or mixed-type dislocations, with an angular orientation coinciding with highsymmetry crystal directions. We detect tens of micrometers long linear lineups of edge dislocations with sizable effects on the local strain states and photoluminescence peak positions. With continuum elastic strain simulations, we demonstrate that the observed patterns of threading dislocations are energetically favorable. We calculated a binding energy per threading dislocation length of $50 \mathrm{meV} / \mu \mathrm{m}$ against gliding of an edge dislocation within a lineup of edge dislocations of parallel Burgers vector orientation.
\end{abstract}

DOI: 10.1103/PhysRevB.75.245213

PACS number(s): 78.55.Cr, 61.72.Ff, 61.72.Bb, 61.72.Hh

\section{INTRODUCTION}

Within the past decade, gallium nitride $(\mathrm{GaN})$ emerged from a poorly understood but promising material system to the well-studied basis of manifold, mainly optical applications. From the very beginning, the impact of threading dislocations (TDs) on device lifetime and optical and transport properties is a central issue in this material system. ${ }^{1}$ Threading dislocations act as centers of nonradiative recombination and limit the efficiency of light-emitting devices. ${ }^{2,3}$ Although heteroepitaxial $\mathrm{GaN}$ with dislocation densities around $10^{9} \mathrm{~cm}^{-2}$ is suitable for the broad field of light-emitting diodes, more demanding applications like laser diodes or highmobility heterostructures need substantially lower defect densities below $10^{7} \mathrm{~cm}^{-2}$, which are provided by GaN bulk crystals grown by hydrogen vapor phase epitaxy (HVPE). The core of TDs can be charged and influence charges in the surrounding material. ${ }^{4}$ However, even without these charges, the mere strain fields of dislocations can disturb the carrier transport in two-dimensional electron gases (2DEGs). ${ }^{5,6}$ This effect can be amplified by the piezoelectric field generated at quantum well interfaces. ${ }^{7}$ Consequently, knowledge about the dislocation and strain distribution is crucial for the fabrication of optoelectronic devices and of 2DEG-based electronic devices. The long-range ordering and alignment of dislocations reported here need to be considered in device manufacturing, especially for relatively small structures like the active region of laser diodes.

The main results are presented in Secs. III-V. First, we demonstrate that the single strain-dipole field of unperturbed edge dislocations is oriented according to the six possible orientations of the Burgers vector given by the hexagonal crystal symmetry (Sec. III). Next we discuss observed lineups of edge dislocations and, by comparing their strain field with continuum elastic calculation, we demonstrate the ordering of the dislocations along the lineups (Sec. IV). We show that the observed patterns are energetically favorable in a continuum elastic description of the interaction of threading dislocations (Sec. V). These central sections are preceded by a description of the bulk GaN samples and experimental procedure (Sec. II) and followed by a discussion (Sec. VI) and summary.

\section{EXPERIMENT}

For our investigations we use commercially available bulk GaN crystals grown by HVPE along the $\langle 0001\rangle$ direction by different manufacturers. Figure 1 represents a typical microphotoluminescence $(\mu \mathrm{PL})$ surface scan of the 0.35 -mm-thick sample selected for this study. The integrated near-band-edge intensity shows dark spots, where TDs penetrate the sample surface. The dislocation density of 4 $\times 10^{6} \mathrm{~cm}^{-2}$ (averaged over all $\mu \mathrm{PL}$ scans at the sample) determined by this means is in good agreement with the maximum density of less than $10^{7} / \mathrm{cm}^{2}$ specified by the manufacturer. The diameter of the dark spots is about $1 \mu \mathrm{m}$ [full width at half maximum (FWHM)], which results from a combination of carrier diffusion and the resolution of the setup $(0.6 \mu \mathrm{m})$. All $\mu \mathrm{PL}$ measurements were performed at a nominal temperature of $6 \mathrm{~K}$ with an excitation wavelength of $334 \mathrm{~nm}$. The experimental setup is identical to the one used in Ref. 8 except for a change of the microscope objective to

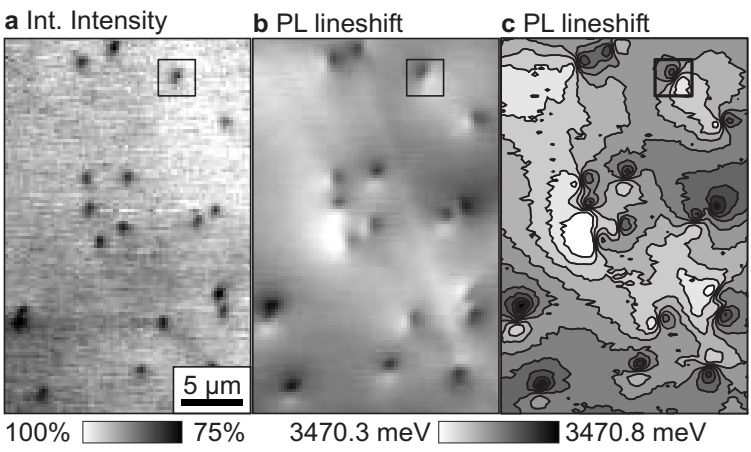

FIG. 1. Microphotoluminescence area scan across the (0001) surface of a bulk GaN sample. (a) Integrated near-band-edge intensity and photoluminescence line shift, represented by the first momentum [(b) in grayscale and (c) as contour plot] of the spectrum. 


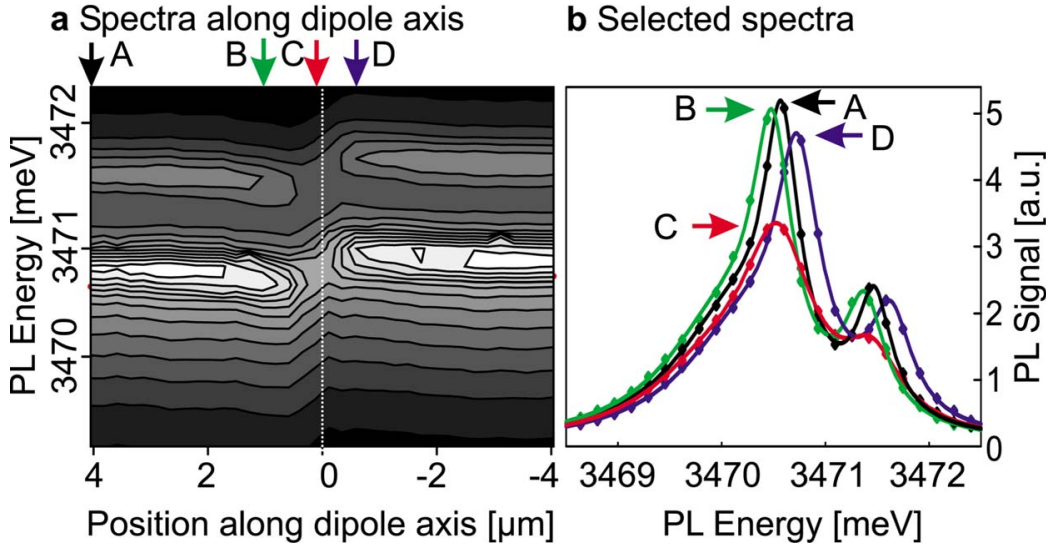

FIG. 2. (Color online) Spectra along one strain dipole induced by an edge- or mixed-type dislocation. (a) shows a contour plot of the photoluminescence spectra along the energy shift dipole main axis of the dislocation marked with a black rectangle in Fig. 1. Spectra at four particular positions are drawn explicitly in (b): A, far away from the dislocation and thus undisturbed; $\mathrm{B}$ and $\mathrm{D}$, with maximum energy shift; and $\mathrm{C}$, at the dislocation core. The marks represent the measured data points, whereas the solid lines are derived from a multiline fit of the spectra with three Lorentzian curves. an Ealing reflective objective (52×, numerical aperture $=0.65)$.

Because of the small linewidth of the donor bound exciton lines $[\sim 1 \mathrm{meV}$ FWHM, see Fig. 2(b)] which dominate the near-band-edge spectrum, we can detect small, strainrelated shifts $\Delta E$ in the photoluminescence (PL) energy. Around each dislocation core, a dipolelike shift with an amplitude of about $\pm 0.1 \mathrm{meV}$ is detected [Figs. 1(b) and 1(c)]. This energy shift is due to elastic lattice deformation ${ }^{8}$ by the strain fields of edge- or mixed-type TDs [see Fig. 3(b)]: Symmetrically at one side of the dislocation the additional lattice plane causes compressive strain, whereas at the opposite side tensile strain is induced.

The measured energy shift $\Delta E$ (which is proportional to the sum of the directional strains in $x$ and $y$ directions ${ }^{9} \varepsilon_{x x}$ $\left.+\varepsilon_{y y}\right)$ is found to be in perfect agreement with an elastic deformation model taking into account the free sample surface and the resolution of the microscope. As described and verified in Ref. 10, we use the first momentum instead of fitting each spectral line for the determination of the energetic shifts $\Delta E$ of the PL position. Figure 2 shows the near-

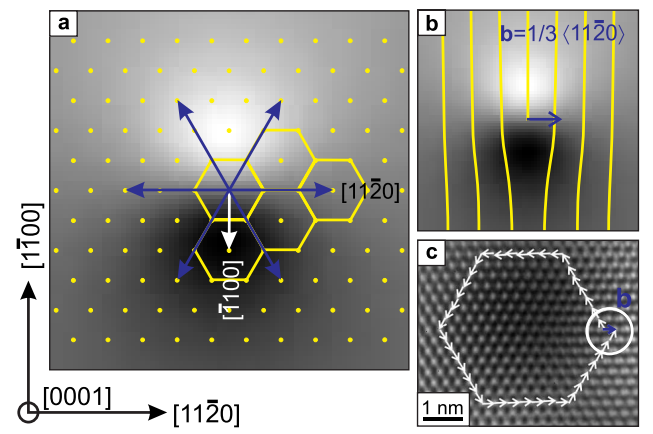

FIG. 3. (Color online) (a) illustrates the possible angular orientation of the Burgers vectors (dark arrows: $\langle 11 \overline{2} 0\rangle$ ) of perfect edge dislocations along the $c$ crystal axis in wurtzite GaN. Dots symbolize the hexagonal lattice sites. The grayscale image schematically shows one possible $\langle\overline{1} 100\rangle$ orientation (light arrow) of the resulting strain dipole (not in scale with the lattice points). In (b) the correlation of crystal planes (lines), Burgers vectors (arrow), and strain dipole (grayscale image, not to scale with crystal planes) is visualized. (c) shows a plan-view HRTEM image of such a dislocation with a $1 / 3[11 \overline{2} 0]$ edge component (determined by a Burgers circuit). band-edge spectra (mainly donor bound exciton lines) along a cut through a typical dislocation along the energy shift dipole. The diminishing and energy shifting of the emission around the dislocation core (at the vertical center of the graph) are clearly visible.

Each dislocation (as seen in Fig. 1), except for very close pairs and few exceptions in other scan areas (not shown here), is associated with a strain dipole and is therefore of edge or mixed type. The energy shift signature of pure edgeand mixed-type dislocations with a corresponding edge component is nearly identical, as the screw component of a dislocation does not cause hydrostatic strain. Therefore it is not possible to distinguish between the two types in $\mu \mathrm{PL}$ measurements. So, in the following, the expression "edge dislocation" is used synonymously for perfect edge- or mixedtype dislocation with an edge component. Pure screw dislocations are rare in these samples, which is in agreement with other investigation techniques such as etch pit density measurements and transmission electron microscopy (TEM).

\section{ALIGNMENT OF STRAIN DIPOLES WITH SIXFOLD CRYSTAL SYMMETRY}

As Fig. 3(a) demonstrates, due to the $120^{\circ}$ in-plane symmetry of wurtzite GaN, there are six possible equivalent directions for the $\langle 11 \overline{2} 0\rangle$ edge dislocation Burgers vectors. Therefore, there are six possible orientations of the associated strain dipole in $\langle 1 \overline{1} 00\rangle$ direction, perpendicular to the Burgers vector [see Fig. 3(b)]. The edge component of a dislocation's Burgers vector can be determined by a Burgers circuit in high-resolution transmission electron microscopy (HRTEM) micrographs [Fig. 3(c)], in which the dislocation core configuration is visible ${ }^{11}$ and the local strain in close neighborhood to the core region can be determined. ${ }^{12}$

In order to determine precisely the orientation of the strain field dipoles, we use a correlation method. A correlation core pattern was generated, which represents the expected PL energy shift caused by the strain of an edge dislocation as measured by a confocal microscope. The pattern was derived from elastic deformation theory convoluted with an $\sim 1 \mu \mathrm{m}$ FWHM two-dimensional (2D) Gaussian function to take into account the microscope resolution. ${ }^{8}$ We generate a complex-valued core pattern by adding the imaginary $i$ times the $90^{\circ}$ rotated core. With this complex core, it is pos- 


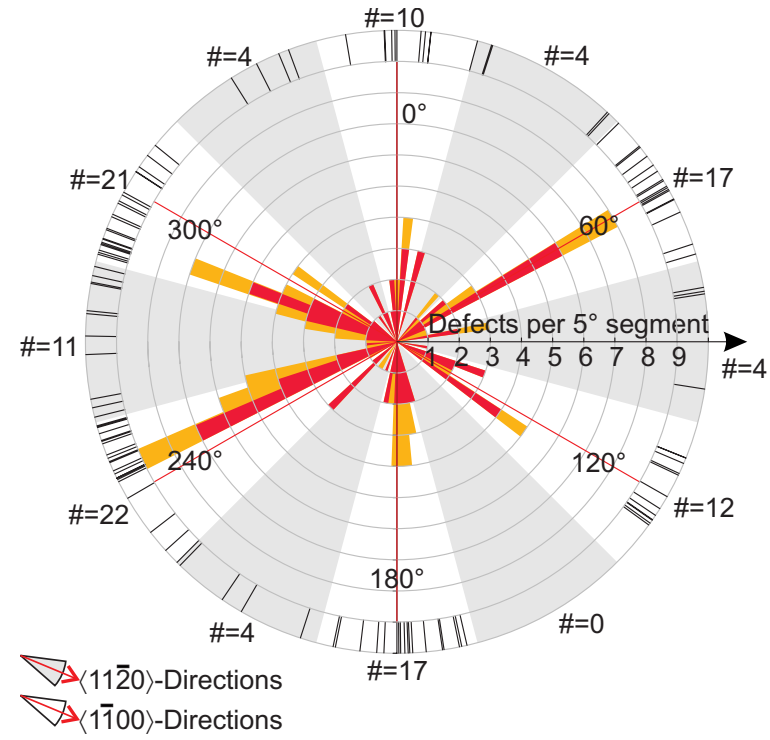

FIG. 4. (Color online) Histogram of the angular dispersion of the stress-related dipoles induced by edge dislocations. The ticks at the outer circle mark the directions of the 126 evaluated dislocation dipoles. The dark and light angular histogram accounts for 84 completely freestanding (undisturbed) dislocations and 42 dislocations which might be slightly influenced by their neighbors, respectively. The full angular circle is divided in $30^{\circ}$ sectors, whereby the numbers outside each sector give the total number of dislocations within. For better visualization, the shaded sectors are centered around the $\langle 11 \overline{2} 0\rangle$ directions, where dislocations should be absent, and the unshaded sectors are centered around the $\langle 1 \overline{1} 00\rangle$ directions (lines), in which one expects the dislocation dipoles.

sible to determine the position and orientation of the dislocation strain pattern simultaneously. The maximum absolute value of a complex-valued 2D correlation marks the center of the energy shift dipole, and the complex number's angular argument at the center is the sought-after dipole angle. For a detailed description of the method, see Ref. 13. This method works in analogy to the phase angle determination in a twophase lock-in amplifier.

We have tested this method for artificially generated, rotated dipole patterns and found an angular resolution better than $\pm 2^{\circ}$. Only if the angle is closer than $10^{\circ}$ to multiples of $90^{\circ}$ will this method tend to assume an exact orientation in $90^{\circ}$ directions due to the ambiguity caused by the limited resolution, and the accuracy gradually drops to $\pm 5^{\circ}$. The core patterns used for the direction detection had a resolution of $0.25 \mu \mathrm{m} /$ pixel (same as the $\mu \mathrm{PL}$ scans) and covered an area of $13 \times 13$ pixels.

As the defect strain fields can overlap and superimpose each other, which disturbs the direction determination, we selected 126 freestanding dislocations from a scan area of $14350 \mu \mathrm{m}^{2}$ on our sample for the histogram shown in Fig. 4. The directional distribution of the edge component of the Burgers vectors is shown in Fig. 4. One can clearly see the sixfold symmetry of the preferential angular orientation, which is exactly what is expected for the hexagonal symmetry of GaN.

From the $\{1 \overline{100}\}$ cleavage planes of the sample, we determined the absolute orientation of the crystal axes in Fig. 4.

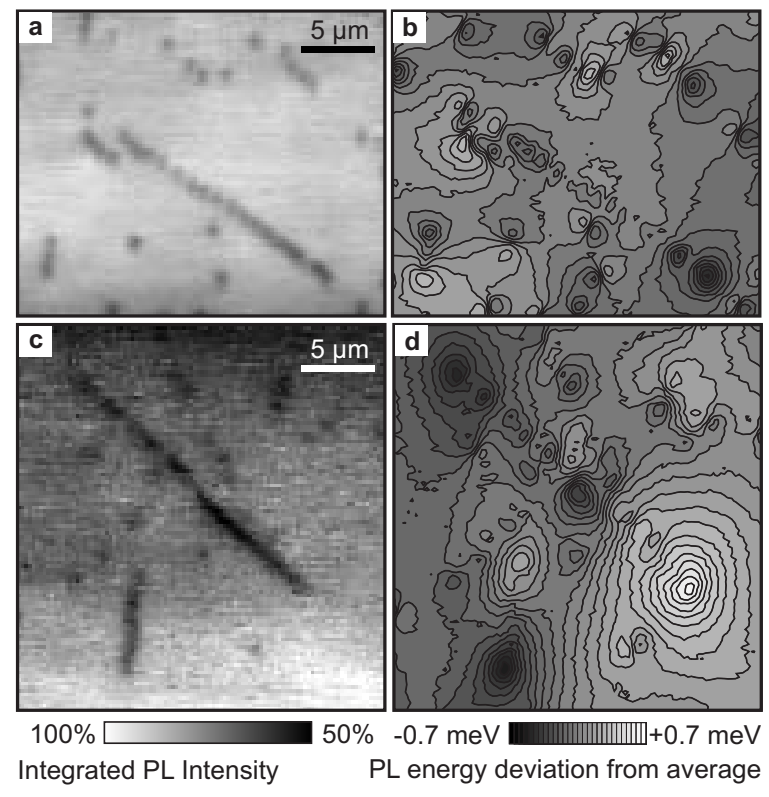

FIG. 5. (a) and (c) show the integrated near-band-edge intensity of area scans at different regions on the investigated sample. The adjacent contour plots [(b) and (d)] depict the energy deviations of the near-band-edge PL from the same region, where every contour level refers to $0.05 \mathrm{meV}$ energy shift.

This orientation was later independently confirmed by x-ray diffraction (XRD) measurements. As a result, we can unambiguously assign the dipolelike strain states to threading edge- or mixed-type dislocations with edge component.

\section{STRAIN FIELDS AND ENERGY SHIFT}

The dislocations tend to form patterns as shown in Fig. 5, where edge dislocations of the same Burgers vector orientation form lines along the $\langle 1 \overline{100}\rangle$ directions. We observe lineups with a length larger than $20 \mu \mathrm{m}$. We detect ten of these structures with an overall length of about $78 \mu \mathrm{m}$ in a total scan area of $1.46 \times 10^{-4} \mathrm{~cm}^{2}$, which corresponds to an overall density of $7 \times 10^{4} \mathrm{~cm}^{-2}$ and a line density of about $50 \mathrm{~cm}^{-1}$.

Next we demonstrate that the strain field causing the energy shift observed for these patterns is given by a linear superposition of the strain fields of a string of edge dislocations of the same orientation. Continuum elastic calculations ${ }^{9}$ are sufficient to describe the strain fields because the distance between individual dislocations is large $(\approx 1 \mu \mathrm{m})$ compared to the dimension of the dislocation core $(\approx 1 \mathrm{~nm})$. We are dealing with edge dislocations threading along the $c$ axis. For an infinite crystal of hexagonal symmetry, the strain field of an edge dislocation with Burgers vector

$$
b=\frac{1}{3}\langle 11 \overline{2} 0\rangle
$$

is given by

$$
\varepsilon_{x x}=\frac{b}{4 \pi} y \frac{\left(3 c_{11}+c_{12}\right) x^{2}+\left(c_{11}-c_{12}\right) y^{2}}{c_{11}\left(x^{2}+y^{2}\right)^{2}},
$$




$$
\begin{gathered}
\varepsilon_{y y}=\frac{b}{4 \pi} y \frac{-\left(c_{11}+3 c_{12}\right) x^{2}+\left(c_{11}-c_{12}\right) y^{2}}{c_{11}\left(x^{2}+y^{2}\right)^{2}}, \\
\varepsilon_{x y}=\frac{b}{2 \pi} \frac{c_{11}+c_{12}}{c_{11}} x \frac{x^{2}-y^{2}}{\left(x^{2}+y^{2}\right)^{2}}, \\
\varepsilon_{z z}=\varepsilon_{x z}=\varepsilon_{y z}=0,
\end{gathered}
$$

in Cartesian coordinates $(x \|\langle 11 \overline{2} 0\rangle$ and $y \|\langle 1 \overline{1} 00\rangle)$. For the calculations, we used a value of the Burgers vector of $b$ $=0.3189 \mathrm{~nm}$. The independent elastic constants $c_{i j}$ for $\mathrm{GaN}$ were taken from Ref. 14:

$$
\begin{aligned}
& c_{11}=390 \mathrm{GPa}, \\
& c_{12}=145 \mathrm{GPa}, \\
& c_{13}=106 \mathrm{GPa}, \\
& c_{33}=398 \mathrm{GPa}, \\
& c_{44}=105 \mathrm{GPa} .
\end{aligned}
$$

The resulting hydrostatic volume change

$$
\varepsilon_{x x}+\varepsilon_{y y}=\frac{b}{4 \pi} \frac{2\left(c_{11}-c_{12}\right)}{c_{11}} \frac{y}{x^{2}+y^{2}}
$$

forms a dipole field. From the strain field, the change in the band-gap energy is calculated ${ }^{15}$ as

$$
E_{g}^{\mathrm{eff}}-E_{g}=\Delta E_{g}=\left(a_{1}+b_{1}\right) \varepsilon_{z z}+\left(a_{2}+b_{2}\right)\left(\varepsilon_{x x}+\varepsilon_{y y}\right),
$$

with the hydrostatic deformation potential given by ${ }^{16}$

$$
\begin{gathered}
a_{1}=-5.55 \mathrm{eV}, \\
a_{2}=-9.38 \mathrm{eV}, \\
b_{1}=-6.61 \mathrm{eV}, \\
b_{2}=3.55 \mathrm{eV} .
\end{gathered}
$$

The strain $\varepsilon_{z z}$ is zero for a Burgers vector in the $a$ - $b$ crystal plane, and thus the energy shift distribution is proportional to the hydrostatic strain dipole given by $\varepsilon_{x x}+\varepsilon_{y y}$ in Eq. (4). Apart from the prefactors, the strain distribution for the given symmetry is identical to that of an edge dislocation in a crystal of cubic symmetry as long as the Burgers vector lies in the anisotropic $a-b$ crystal plane.

At the surface the crystal lattice relaxes in the $z$ direction and therefore $\varepsilon_{z z}$ does not vanish. We simulated the effect of this surface term on the energy landscape in a threedimensional simulation of a semi-infinite crystal. ${ }^{8}$ For the penetration depth of the exciting laser at $334 \mathrm{~nm}$, the limited probing volume reduces the observed energy shift by a factor of approximately 2 with respect to the bulk value. This factor depends on the fraction of the near-surface probing volume to the total probing volume, and thus, on the penetration depth of the exciting beam. (a)

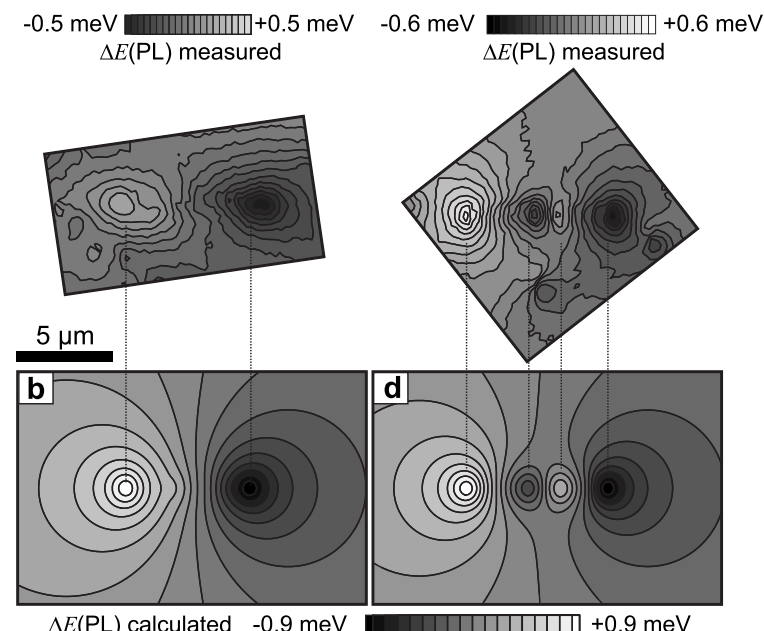

FIG. 6. Measured [(a) and (c)] and calculated [(b) and (d)] energy shifts of the donor bound exciton caused by arrays of edge dislocations. The pattern shown in (a) corresponds to the lineup in the lower left corner of Fig. 5(c); the one shown in (b) is from a different scan area not included in Fig. 5.

The energy shift caused by an ensemble of edge dislocations is calculated by linear superposition of the individual strain fields. From the resulting hydrostatic strain, the energy shift is calculated via Eq. (5). The density plots in Fig. 6 show the observed and simulated energy landscapes for two dislocation patterns: One is a linear array of approximately equidistant edge dislocations [Figs. 6(a) and 6(b)]; the other an intermitted linear array with a more complicated energy pattern [Figs. 6(c) and 6(d)]. Corresponding cuts along the central axis of the dislocation lineups are plotted in Fig. 7. Both figures together demonstrate that the shape of the energy shift can be modeled in the continuum elastic approach. This comparison also shows that all edge dislocations forming the individual lineups have the same orientation, with the Burgers vector perpendicular to the line connecting the edge dislocations. In this configuration the strain fields add up at the end of the lineups, relaying strain over distances up to several $10 \mu \mathrm{m}$.

Figure 8 shows cuts through the lineup main axis in the PL energy landscape for different patterns ranging, from single edge dislocations to a lineup of 19 edge dislocations. It must be mentioned that the real PL energy shift (and thus material strain) very near the dislocation cores $(<1 \mu \mathrm{m})$, which are actually considerably higher, cannot be measured directly by $\mu \mathrm{PL}$ as it is masked by the smoothing effect of the microscope resolution. However, for distances of more than $1 \mu \mathrm{m}$ from the TD core, the confocal measurements represent the strain landscape accurately. The individual dislocation position can be determined either directly from the dark spots in the intensity distribution or from a comparison of the calculated and measured energy shift profiles. The average distance of dislocations forming the lineups is about $1 \mu \mathrm{m}$. Despite this rather long distance, for interacting threading dislocations the linear dislocation aggregates represent an energetically favorable configuration, as will be shown in the next section. Since all strain dipoles in a dislo- 

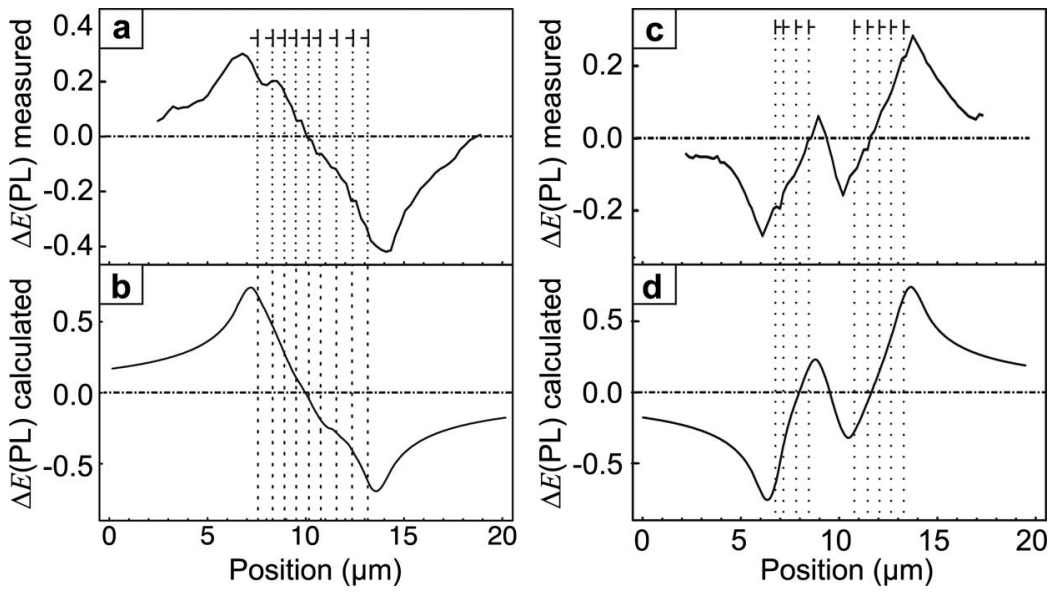

FIG. 7. Cut along the line connecting the dislocations in Fig. 6. (a)-(d) correspond to Figs. 6(a)-6(d), respectively. The dotted lines mark the position of the individual dislocations used to simulate the energy shift patterns.

cation lineup have the same orientation, the lineups can build up considerable stress at the end of a line. As Figs. 8(a)-8(d) show, the built-up strain can cause energy variations in the range of $1 \mathrm{meV}$ on a spatial scale of several micrometers, which is four times as much as a single edge dislocation inflicts [Fig. 8(e)]. The strain fields of more than a dozen dislocations add up and form large areas of enhanced strain at the ends as well as almost completely relaxed material parallel to the lineup.

\section{DIRECTIONAL AND SPATIAL ORDERING}

Here, we demonstrate that the observed patterns of edge dislocations are energetically stable within the continuum elastic model. In particular, we determined the energetic stability of a defect arrangement against glide or climb of the threading dislocations by a calculation of the energy with dependence on the position of one moving dislocation. To do so, the strain of a pattern of dislocations as described in the

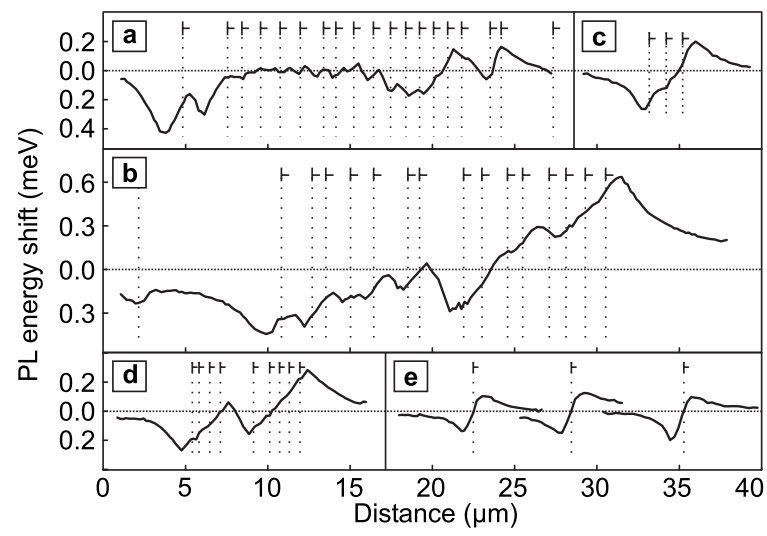

FIG. 8. (a)-(d) show linear cuts through the PL energy landscape along dislocation lineups. (a) and (b) correspond to the dominant lineups in Figs. 5(b) and 5(d), respectively. The dotted vertical lines with the attached dislocation marks indicate the assumed position of the single edge dislocations judging from the PL intensity. (c) and (d) show similar cuts through different dislocation lineups (not shown in Fig. 5) and (e) displays cuts through three freestanding and thus undisturbed dislocations along the main strain-dipole axis for comparison. preceding section was calculated on a $2 \mathrm{D}$ grid of at least $10 \times 10 \mu \mathrm{m}^{2}$ with a point distance of $0.025 \mu \mathrm{m}$. As the dislocation core represents a numeric singularity and the core region is of no interest here anyway, we placed the dislocation core in between the grid points. Then the free energy of the strain field was calculated by summation over the local energy: ${ }^{9}$

$$
\begin{aligned}
F= & c_{11}\left(\varepsilon_{11}^{2}+2 \varepsilon_{12}^{2}+\varepsilon_{22}^{2}\right)+2 c_{12}\left(\varepsilon_{11} \varepsilon_{22}-\varepsilon_{12}^{2}\right) \\
& +2 c_{13} \varepsilon_{33}\left(\varepsilon_{11}+\varepsilon_{22}\right)+c_{33} \varepsilon_{33}^{2}+4 c_{44}\left(\varepsilon_{13}^{2}+\varepsilon_{23}^{2}\right) .
\end{aligned}
$$

For the present situation, only strain components within the $x-y$ plane are nonzero, and so the second line of Eq. (7) becomes zero.

The potential of one dislocation in the strain field of another dislocation of the same Burgers vector orientation is plotted in Fig. 9(a). The energy in the 2D simulation is given per length of the dislocation. In this configuration, the two dislocations generally repel each other. In Fig. 9(b), the potential along the vertical dashed line in Fig. 9(a) is plotted. A motion along this line corresponds to gliding of the edge dislocation. This potential has a local minimum when both dislocations are horizontally aligned. The depth of the local minimum can be considered as binding energy for this configuration. This binding energy slowly decreases but still has a large value of more than $15 \mathrm{meV} / \mu \mathrm{m}$ for a distance between the dislocations of $1 \mu \mathrm{m}$ and larger. If there were climb processes at work, the dislocation alignments would not be stable as the two dislocations repel each other in climb direction.

Two dislocations of opposite Burgers vector orientation attract each other. They can form a dislocation loop and annihilate each other.

A dislocation within a lineup of several dislocations of equal Burgers vector orientation is stable against motion in all directions. The potential of this configuration is shown in Fig. 10, where one dislocation is moved in the strain field generated by four dislocations at fixed positions. The potential has a local minimum in the center between the other dislocations. The binding energy against gliding is consequently positive and with $50 \mathrm{meV} / \mu \mathrm{m}$ more than three times as large as in the case of two dislocations. The potential along the line of dislocations also shows a minimum at the 

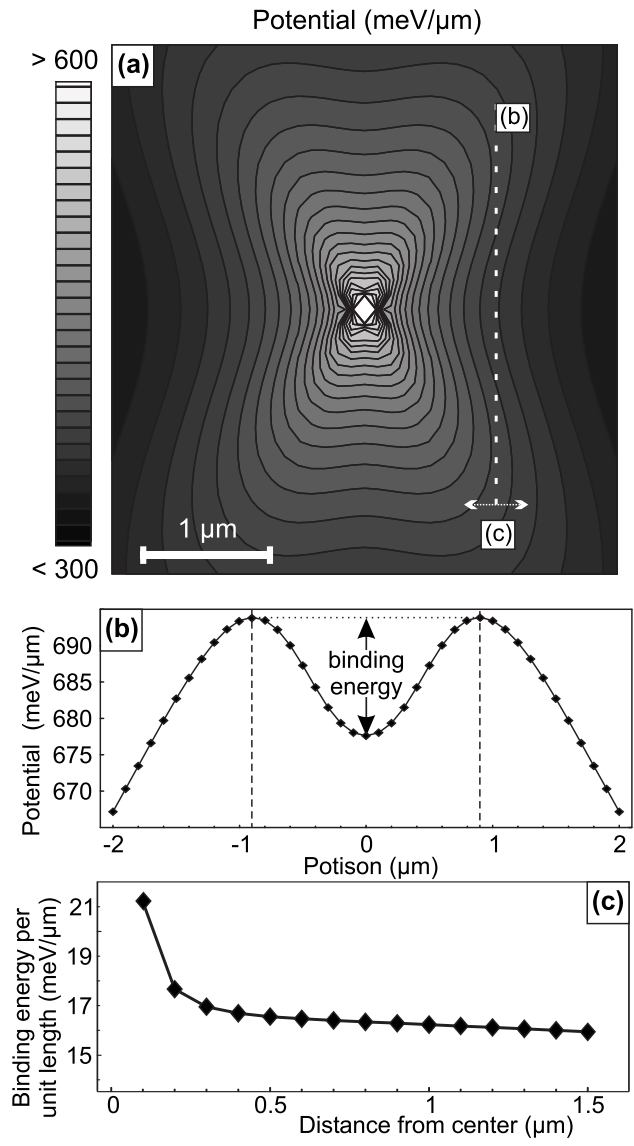

FIG. 9. (a) Calculated potential of an edge dislocation in the strain field of an edge dislocation of the same Burgers vector orientation placed in the center. (b) Potential along the vertical dashed line showing the local minimum for gliding. (c) Binding energy for gliding as function of the distance of the gliding plane (dashed line) from the fixed edge dislocation in the center.

center. In this model, a lineup of edge dislocations can be dissolved only at the ends by a climb process of the outermost edge dislocations.

\section{DISCUSSION}

First, we observe a sixfold symmetry in the orientation of strain dipoles caused by single edge dislocations. While it is obvious that the Burgers vector has one of six possible directions $b=1 / 3\langle 11 \overline{2} 0\rangle$ according to the hexagonal crystal symmetry, it is still astonishing that this symmetry can be observed on a macroscopic spatial scale three orders of magnitude larger than the dimension of the dislocation core. This is the case only if the crystal is unperturbed on this length scale, i.e., if the dislocation density is low enough. While we were able to measure the strain dipoles on several different bulk GaN samples grown by different groups, we could prove the sixfold orientation only for the sample with the lowest dislocation density. For all other samples from different sources, the number of solitary dislocations in a single scan was too low to produce a significant statistic comparable to Fig. 4.
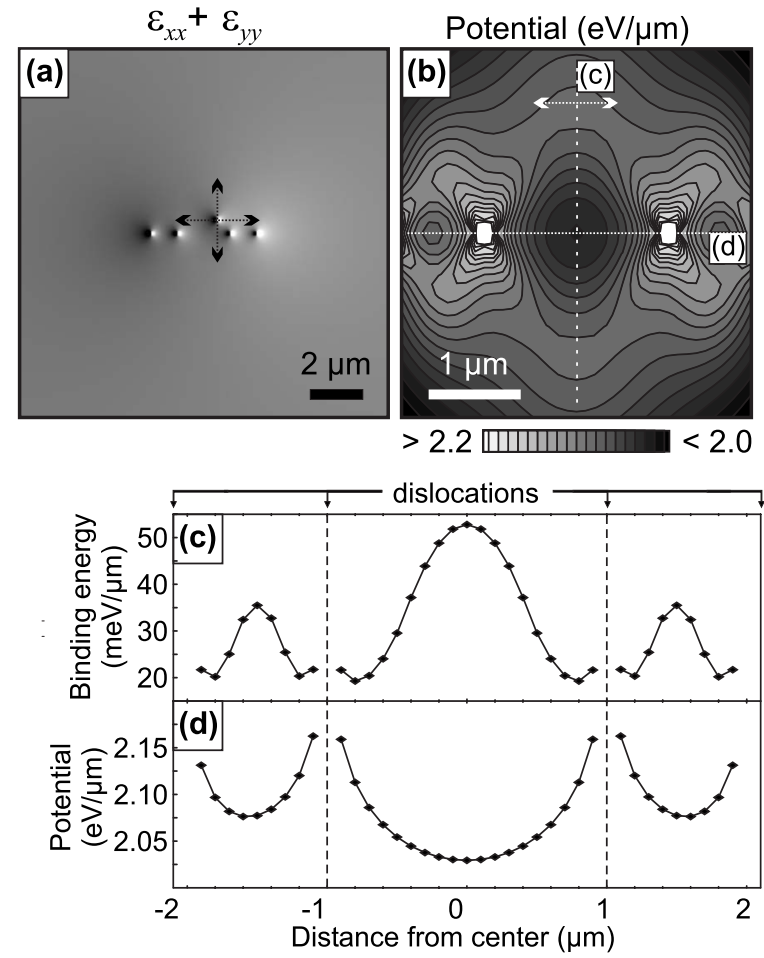

FIG. 10. (a) Hydrostatic strain field for a pattern of four fixed and one movable edge dislocations of identical Burgers vector orientation. (b) Potential as function of the position of the movable dislocation. (c) Binding energy for gliding along the vertical dashed line in the center. (d) Potential along the horizontal line through the four fixed edge dislocations.

The sample shows patterns of single edge dislocations, where from a few up to 20 individual dislocations line up. Within one lineup the Burgers vectors of every dislocation have the same orientation perpendicular to the line. As a consequence, the orientation of these lineups also shows a sixfold symmetry. It is not unlikely that the dislocations emanate from crystal grain boundaries in the initial growth layers. TEM images of GaN samples with high dislocation density suggest an ordering of dislocations along grain boundaries (see, e.g., Ref. 17). Yet when compared to those samples, the distance between threading dislocations in our sample is huge. The continuum elastic calculations still show that the lineups are energetically favorable. With a binding energy per dislocation length of several ten $\mathrm{meV} / \mu \mathrm{m}$, the total binding energy of dislocations running parallel for a few micrometers becomes so large that this configuration could be stable even at growth temperatures. An annihilation by recombination of two dislocations within one pattern is impossible because of the identical orientation of the Burgers vectors. So, independent of their formation, either at the initial growth step or through accumulation during the growth of the several $100-\mu \mathrm{m}$-thick GaN layer, these lineups are stable patterns. This will affect the further reduction of dislocation during the growth of thick GaN layers and possibly limit the achievable threading dislocation density.

\section{SUMMARY}

In summary, we verify the expected dipolelike energy shift around single edge (or mixed type) dislocations on a 
micrometer length scale. For the investigated sample with its very low threading dislocation density, we were able to substantiate the discrete orientation of the dislocation dipoles along the six $\langle 1 \overline{1} 00\rangle$ crystal axes, which is in perfect agreement with the orientation of edge dislocations with Burgers vectors $b=1 / 3\langle 11 \overline{2} 0\rangle$ given by the hexagonal lattice symmetry. The absolute orientation of the dislocations matches the expected directions derived from the cleavage plane orientations and measured by XRD. Furthermore, we encountered long-range dislocation ordering over tens of micrometers with an average next-neighbor distance of about $1 \mu \mathrm{m}$. At the ends of these linear clusters, strain is accumulated, with great impact on the surrounding material. The PL emission of tens of square micrometers can be shifted up to $1 \mathrm{meV}$ in energy.

We demonstrate that the observed strain patterns can be described in a continuum elastic model as a linear superpo- sition of the strain fields of the individual threading dislocations. We determine the stability of the dislocation patterns against glide and climb of individual dislocations. From the free energy of the strain field, the binding energy per dislocation length against gliding of a single dislocation within a lineup of dislocations of parallel Burgers vectors was calculated to be $50 \mathrm{meV} / \mu \mathrm{m}$. As a result, the observed patterns of dislocations are energetically favorable in the continuum elastic model.

\section{ACKNOWLEDGMENTS}

We thank Karl Engl for the TEM image and fruitful discussions, and Osram Opto Semiconductors $\mathrm{GmbH}$ for their support of our work. We acknowledge funding by the German Federal Ministry of Education and Research (BMBF) grant Nanolux. U.T.S. acknowledges support from the Japan Society for the Promotion of Science (JSPS).
*Electronic address: ulrich.schwarz@ physik.uni-regensburg.de

${ }^{1}$ I. Akasaki and H. Amano, Jpn. J. Appl. Phys., Part 1 45, 9001 (2007).

${ }^{2}$ T. Sugahara, H. Sato, M. Hao, Y. Naoi, S. Kurai, S. Tottori, K. Yamashita, K. Nishino, L. Romano, and S. Sakai, Jpn. J. Appl. Phys., Part 2 37, L398 (1998).

${ }^{3}$ D. Cherns, S. J. Henley, and F. A. Ponce, Appl. Phys. Lett. 78, 2691 (2001).

${ }^{4}$ A. Krtschil, A. Dadgar, and A. Krost, Appl. Phys. Lett. 82, 2263 (2003).

${ }^{5}$ D. Jena and U. K. Mishra, Appl. Phys. Lett. 80, 64 (2002).

${ }^{6}$ W. Knap, C. Skierbiszewski, K. Dybko, J. Lusakowski, M. Siekacz, I. Grzegory, and S. Porowski, J. Cryst. Growth 281, 194 (2005).

${ }^{7}$ C. Shi, P. M. Asbeck, and E. T. Yu, Appl. Phys. Lett. 74, 573 (1999).

${ }^{8}$ N. Gmeinwieser, P. Gottfriedsen, U. T. Schwarz, W. Wegscheider, R. Clos, A. Krtschil, A. Krost, A. Weimar, G. Brüderl, A. Lell, and V. Härle, J. Appl. Phys. 98, 116102 (2005).
${ }^{9}$ J. P. Hirth and J. Lothe, Theory of Dislocations (McGraw-Hill, New York, 1968).

${ }^{10}$ N. Gmeinwieser, K. Engl, P. Gottfriedsen, U. T. Schwarz, J. Zweck, W. Wegscheider, S. Miller, H.-J. Lugauer, A. Leber, A. Weimar, A. Lell, and V. Härle, J. Appl. Phys. 96, 3666 (2004).

${ }^{11}$ S. Kret, J. Chen, P. Ruterana, and G. Nouet, Inst. Phys. Conf. Ser. 196, 319 (2001)

${ }^{12}$ S. Kret, P. Dluzewski, G. Maciejewski, V. Potin, J. Chen, P. Ruterana, and G. Nouet, Diamond Relat. Mater. 11, 910 (2002).

${ }^{13}$ N. Gmeinwieser and U. T. Schwarz, Phys. Status Solidi B 244, 1857 (2007).

${ }^{14}$ I. Vurgaftman and J. R. Meyer, J. Appl. Phys. 94, 3675 (2003).

${ }^{15}$ J.-M. Wagner and F. Bechstedt, Phys. Rev. B 66, 115202 (2002).

${ }^{16}$ J. A. Majewski, M. Städele, and P. Vogl, MRS Internet J. Nitride Semicond. Res. 1, 30 (1996).

${ }^{17}$ C. Trager-Cowan, F. Sweeney, P. W. Trimby, A. P. Day, A. Gholinia, N.-H. Schmidt, P. J. Parbrook, A. J. Wilkinson, and I. M. Watson, Phys. Rev. B 75, 085301 (2007). 